\title{
A Bivariate Replacement Policy for an Extreme Shock Maintenance Model Under QUASI Renewal Process
}

\author{
Rizwan. U, Govindaraju. P and R. Usman Baig \\ Department of Mathematics, Islamiah College, Vaniyambadi, India. \\ e-mail: rizwan.phd7@gmail.com
}

\begin{abstract}
Considering an extreme shock maintenance model for a degenerative simple repairable system, explicit expressions for the long-run average cost under the bivariate replacement policy $(T, N)$ has been obtained. Existence of optimal value of $(T, N)$ has been deduced.
\end{abstract}

Keywords: Quasi Renewal Process, Replacement Policy, Renewal Reward Process, Shock models.

AMS Subject Classification: 60K10, 90B25.

\section{INTRODUCTION}

Chen and $\mathrm{Li}$ (2008) have considered an extreme shock model and studied the maintenance problem under $N$ policy. Considering the extreme shock model, we study the maintenance problem using the bivariate replacement policy. We also show that the bivariate optimal replacement policy $(T, N)^{*}$ is better than the univariate optimal replacement $N^{*}$ and $T^{*}$ policies.

The rest of the paper is organized as follows. In section 2, we present an extreme shock model for the maintenance problem of a repairable system. In section 3, explicit expressions for the long-run average cost under a bivariate replacement policy $(T, N)$ is derived. Existence of the optimality under the bivariate replacement policy is deduced. Finally, conclusion is given in section 4 .

\section{MODEL DESCRIPTION}

We make the following assumptions about the model for a simple degenerative reparable system subject to shocks.

2.1 At time $t=0$, a new system is installed. Whenever the system fails, it will be repaired. The system will be replaced by an identical new one, some times later.

2.2 Once the system is operating, the shocks from the environment arrive according to a renewal process. Let $X_{n i}, i=1,2, \ldots$ be the intervals between the $(i-1)$-st and $i$-th shock, after the $(n-1)$-st repair. Let $E\left(X_{11}\right)=\lambda$. Assume that $X_{n i}, i=1,2, \ldots$, are iid sequences, for all $n \in N$.

2.3 Let $Y_{n i}, i=1,2, \ldots$ be the sequence of the amount of shock damage produced by the $i$ th shock, after the $(n-1)$-st repair. Let $E\left(Y_{11}\right)=\mu$. Then $\left\{Y_{n i}, i=1,2, \ldots\right\}$ are iid sequences, for all $n \in N$. If the system fails, it is closed, so that the random shocks have no effect on the system during the repair time. In the $n$-th operating stage, that is, after the $(n-1)$-st repair, the system will fail, if the amount of the shock damage first exceed $a^{n-1} M$, where $0<a \leq 1$ and $M$ is a positive constant.

2.4 Let $Z_{n}, n=1,2, \ldots$ be the repair time after the $n$-th repair and $Z_{n}, \quad n=1,2, \ldots$ constitute a non decreasing quasi renewal process with $E\left(Z_{1}\right)=\delta$ and ratio $b$, such that $0<b<1$. $N_{n}(t)$ is the counting process denoting the number of shocks after the $(n-1)$-st repair. It is clear that $E\left(Z_{n}\right)=\delta \beta^{n-1}$.

2.5 Let $r$ be the reward rate per unit time of the system when it is operating and $c$ be the repair cost rate per unit time of the system and the replacement cost is $R$. The replacement time is a random variable $Z$ with $E(Z)=\tau$.

2.6 The sequences $\left\{X_{n i}, i=1,2, \ldots\right\},\left\{Y_{n i}, i=\right.$ $1,2, \ldots\}, \quad\left\{Z_{n}, n=1,2, \ldots\right\}$ and $Z$ are independent.

2.7 The replacement policy $(T, N)$ is adapted.

\section{THE BIVARIATE REPLACEMENT POLICY $(\mathbf{T}, \mathbf{N})$}

In this section, we study an extreme shock model for the maintenance problem of a simple repairable system under $(T, N)$ policy. Let

$$
L_{n}=\min \left\{l ; \quad Y_{n l}>a^{n-1} M\right\}
$$

and $\quad W_{n}=\sum_{i=1}^{L_{n}} X_{n i}$.

Thus, $L_{n}$ is the number of shocks until the first deadly shock occurred following the $(n-1)$-st failure and $L_{n}$ has a geometric distribution with $P\left\{L_{n}=k\right\}=$ $p_{n} q_{n}{ }^{k-1}, \quad k=1,2, \ldots$, where $p_{n}=P\left\{Y_{n l}>a^{n-1} M\right\}$ and $q_{n}=1-p_{n}$. We have $E\left(L_{n}\right)=\frac{1}{p_{n}}$. Since $\left\{X_{n i}, i=1,2, \ldots\right\} \quad$ and $\quad\left\{Y_{n i}, i=1,2, \ldots\right\} \quad$ are independent, it is clear that $L_{n}$ and $\left\{X_{n i}, i=1,2, \ldots\right\}$ are independent. Now 


\section{Available online at www.ijrat.org}

$$
\begin{aligned}
E\left(W_{n}\right) & =E\left(\sum_{i=1}^{L_{n}} X_{n i}\right) \\
& =E\left(L_{n}\right) E\left(X_{n 1}\right) \\
& =\frac{\lambda}{p_{n}} .
\end{aligned}
$$

The distribution function of $W_{n}$ is $F_{n}(\cdot)$.

The working age $T$ of the system at time $t$ is the cumulative life-time given by

$$
T(t)=\left\{\begin{array}{l}
t-V_{n}, \quad U_{n}+V_{n} \leq t<U_{n+1}+V_{n} \\
U_{n+1}, \quad U_{n+1}+V_{n} \leq t<U_{n+1}+V_{n+1}
\end{array}\right.
$$

where $U_{n}=\sum_{i=1}^{n} W_{i}$ and $V_{n}=\sum_{i=1}^{n} Z_{i}$ and $U_{0}=$ $V_{0}=0$.

Let $T_{1}$ be the first replacement time; in general for $n=2,3, \ldots$, let $T_{n}$ be the time between the $(n-1)$-st replacement and the $n$-th replacement. Thus the sequence $\left\{T_{n}, n=1,2, \ldots\right\}$ forms a renewal process. A cycle is completed, if a replacement is done. A cycle is actually the time interval between the installation of the system and the first replacement or the time interval between two consecutive replacements. Finally, the successive cycles together with the cost incurred in each cycle will constitute a renewal reward process. The length of the cycle under the replacement policy $(T, N)$ is

$$
\begin{aligned}
W=\left(T+\sum_{n=1}^{\eta} Z_{n}\right) \chi_{\left(U_{N}>T\right)} & \\
& +\left(\sum_{n=1}^{N} W_{n}+\sum_{n=1}^{N-1} Z_{n}\right) \chi_{\left(U_{N} \leq T\right)}+Z,
\end{aligned}
$$

where $\eta=0,1,2, \ldots, N-1$ is the number of failures before the working age of the system exceeds $T$ and $\chi_{(A)}$ denotes the indicator function. The expected length of the cycle is

$$
\begin{aligned}
E(W)= & E\left[\left(T+\sum_{n=1}^{\eta} Z_{n}\right) \chi_{\left(U_{N}>T\right)}\right] \\
& +E\left[\left(\sum_{n=1}^{N} W_{n}+\sum_{n=1}^{N-1} Z_{n}\right) \chi_{\left(U_{N} \leq T\right)}\right] \\
& +E(Z) \\
= & E\left[T \chi_{\left(U_{N}>T\right)}\right]+E\left[\left(\sum_{n=1}^{\eta} Z_{n}\right) \chi_{\left(U_{N}>T\right)}\right] \\
+ & E\left\{E \left[\left(\sum_{n=1}^{N} W_{n}+\sum_{n=1}^{N-1} Z_{n}\right) \chi_{\left(U_{N} \leq T\right)} \mid U_{N}=\right.\right. \\
& u]\}+E(Z) \\
= & T \bar{F}_{N}(T)+\sum_{n=1}^{N-1}\left(\delta \beta^{n-1}\right) E\left[\chi_{\left(U_{n} \leq T<U_{N}\right)}\right]
\end{aligned}
$$

$$
\begin{gathered}
+\int_{0}^{T} E\left(\sum_{n=1}^{N} W_{n}\right) u d F_{N}(u)+ \\
\int_{0}^{T}\left(\sum_{n=1}^{N-1} E\left(Z_{n}\right)\right) d F_{N}(u)+\tau \\
=T \bar{F}_{N}(T)+\sum_{n=1}^{N-1} \quad \delta \beta^{n-1} P\left(U_{n} \leq T<U_{N}\right) \\
+\sum_{n=1}^{N} \frac{\lambda}{p_{n}} \int_{0}^{T} u d F_{N}(u)+ \\
\sum_{n=1}^{N-1}\left(\delta \beta^{n-1}\right) F_{N}(T)+\tau \\
=T \bar{F}_{N}(T)+\sum_{n=1}^{N-1}\left(\delta \beta^{n-1}\right)\left[F_{n}(T)-F_{N}(T)\right] \\
+\sum_{n=1}^{N} \frac{\lambda}{p_{n}} \int_{0}^{T} u d F_{N}(u)+ \\
\sum_{n=1}^{N-1}\left(\delta \beta^{n-1}\right) F_{N}(T)+\tau \\
=T \bar{F}_{N}(T)+\delta \sum_{n=1}^{N-1} F_{n}(T) \beta^{n-1}+ \\
\sum_{n=1}^{N} \frac{\lambda}{p_{n}} \int_{0}^{T} u d F_{N}(u)+\tau
\end{gathered}
$$

Let $\mathcal{C}(T, N)$ be the long-run average cost per unit time under the bivariate replacement policy $(T, N)$. By the renewal reward theorem, the long-run average cost per unit time under the replacement policy- $(T, N)$ is given by

$$
\begin{aligned}
& \mathcal{C}(T, N) \\
& =\frac{\text { the expected cost incurred in a cycle }}{\text { the expected length of a cycle }} \\
& =\frac{\left[\begin{array}{l}
E\left\{\left(c \sum_{n=1}^{\eta} Z_{n}-r T\right) \chi_{\left(U_{N}>T\right)}\right\}+c_{p} E(Z) \\
+E\left\{\left(c \sum_{n=1}^{N-1} Z_{n}-r \sum_{n=1}^{N} W_{n}\right) \chi_{\left(U_{N} \leq T\right)}\right\}+R
\end{array}\right]}{E(W)} .
\end{aligned}
$$

Using the equation (3.1) in equation (3.2), we obtain the following result.

Theorem 3.1 For the model described in Section 2, under the assumptions 2.1 to 2.7, the long-run average cost per unit time under the bivariate replacement policy $(T, N)$ for a simple degenerative repairable system is given by

$$
\begin{aligned}
& \mathcal{C}(T, N) \\
& =\frac{c \delta \sum_{n=1}^{N-1} F_{n}(T) \beta^{n-1}+c_{p} \tau+R-r T \bar{F}_{N}(T)-r \sum_{n=1}^{N} \frac{\lambda}{p_{n}} \int_{0}^{T} u d F_{N}(u}{T \bar{F}_{N}(T)+\delta \sum_{n=1}^{N-1} F_{n}(T) \beta^{n-1}+\sum_{n=1}^{N} \frac{\lambda}{p_{n}} \int_{0}^{T} u d F_{N}(u)+\tau}
\end{aligned}
$$

\section{Deductions}

The long-run average cost $\mathcal{C}(T, N)$ is a bivariate function in $T$ and $N$. Obviously, when $N$ is fixed, $\mathcal{C}(T, N)$ is a function of $T$. For fixed $N=m$, it can be written as

$$
\mathcal{C}(T, N)=C_{m}(T), \quad m=1,2, \ldots .
$$


Thus, for a fixed $m$, we can find $T_{m}^{*}$ by analytical or numerical methods such that $C_{m}\left(T_{m}^{*}\right)$ is minimized. That is, when $N=1,2, \ldots, m, \ldots$, we can find $T_{1}^{*}, T_{2}^{*}, T_{3}^{*}, \ldots, T_{m}^{*}, \ldots$, respectively, such that the corresponding $C_{1}\left(T_{1}^{*}\right), C_{2}\left(T_{2}^{*}\right), \ldots, C_{m}\left(T_{m}^{*}\right), \ldots \quad$ are minimized. Because the total life-time of a multistate degenerative system is limited, the minimum of the long-run average cost per unit time exists. So we can determine the minimum of the long-run average cost per unit time based on $C_{1}\left(T_{1}^{*}\right), C_{2}\left(T_{2}^{*}\right), \ldots, C_{m}\left(T_{m}^{*}\right), \ldots$.

Then, if the minimum is denoted by $C_{n}\left(T_{n}^{*}\right)$, we obtain the bivariate optimal replacement policy $(T, N)^{*}$ such that

$$
\begin{aligned}
& \mathcal{C}\left((T, N)^{*}\right)=\min _{N} C_{n}\left(T_{n}^{*}\right) \\
& =\min _{N}\left[\min _{T} C(T, N)\right] \\
& \quad \leq \min _{N} C(\infty, N) \equiv C\left(N^{*}\right)
\end{aligned}
$$

the optimal policy $(T, N)^{*}$ is better than the optimal policy $N^{*}$. Moreover, under some mild conditions, Stadje and Zuckerman (1990) showed that an optimal replacement policy $N^{*}$ is better than the optimal policy $T^{*}$. So under the same conditions, an optimal policy $(T, N)^{*}$ is better than the optimal replacement policies $N^{*}$ and $T^{*}$.

\section{CONCLUSION}

Considering an extreme shock maintenance model for a degenerative simple repairable system, explicit expressions for the long-run average cost under the bivariate replacement policy $(T, N)$ has been obtained. Existence of optimal value of $(T, N)$ has been deduced.

\section{REFERENCES}

[1] Badiya, F. G. and Berrade, M. D., (2009), Optimum Maintenance Policy of a Periodically Inspected System under Imperfect Repair, Advances in Operations Research, 1-13

[2] Bai, J.M., Li, Z.H. and Kong, X.B., (2006), Generalized Shock Models based on a Cluster Point Process, IEEE Transactions on Reliability, 55(3), 542-

[3] Barlow, R.E. and Hunter, L.C., (1959), Optimum Preventive Maintenance Policies, Operations Research, 8, 90-100

[4] Braun, W.J, Li Wei and Zhao, Y.Q., (2005), Properties of the Geometric and Related Process, Naval Research Logistics, 52, 607-617

[5] J. and Li, Z.H., (2008), An Extended Extreme Shock Maintenance Model for a Deteriorating System, Reliability Engineering and System Safety, 93, 1123-1129.

[6] Finkelstein, M. and Zarudnij, V., (2001), A Shock Process with a Non-cumulative Damage, Reliability Engineering and System Safety, 71(1), 103-107.

[7] Govindaraju, P., Rizwan, U. and Thagaraj, V., (2011), An Extreme Shock Maintenance Model under a Bivariate Reaplacement Policy, Research Methods in Mathematical Sciences, 1-10.

[8] Lam, Y. and Zhang, Y.L., (2004), A Shock Model for the Maintenance Problem of a Repairable System, Computers and Operations Research, 31, 1807-1820

[9] Lam, Y., (2007), The Geometric Process and its Applications, World Scientific Publishing Co. Pte. Ltd.

[10] Rizwan, U., Syed Tahir Hussainy and Manigandan, J., (2017), An Optimal Bivariate Replacement Policy for a Multistate Degenerative System with an Extreme Shock, International Journal of Current Research in Science and Technology, 3(6), 1-9

[11] Rizwan, U., Govindaraju, P. and Babu, D., (2018), An Extended Extreme Shock Maintenance Model for a Deteriorating System Under Partial Product Process, International Journal of Applied Engineering Research, 13 (16), 12597-12601

[12] Ross, S.M., (1997), Introduction to Probability Modelling, Academic Press, New York.

[13] Sarada, G. and Rangan, A., (2000), Maintenance of Deteriorating Systems Subject to Shocks, Opsearch, 37 (3), 259-266.

[14] Shanthikumar, J.G. and Sumita, U., (1983), General Shock Models Associated with Correlated Renewal Sequences, Journal of Applied Probability, 20 (3), 600-614.

[15] Taylor, H.M. and Karlin, S., (2001), An Introduction to Stochastic Modelling, Academic Press, New York.

[16] Zhi Sheng Ye, Tang, L.C. and Hai Yan Xu, (2011), A Distribution-based Systems Reliability Model under Extreme Shocks and Natural Degradation, IEEE Transactions on Reliability, 60, 246-256. 